\title{
Análise Exploratória de Dados Industriais da Fabricação de Placas de Média Densidade
}

\author{
Thadeu Pezzin Melo ${ }^{1}$, Karin Satie Komati ${ }^{1}$ \\ ${ }^{1}$ Instituto Federal do Espírito Santo - IFES Campus Serra \\ ES-010 KM 65 - 29.173-083 - Manguinhos - Serra - ES - Brasil \\ tpezzin@gmail.com, kkomati@ifes.edu.br
}

\begin{abstract}
This article describes the exploratory data analysis of the MDF board manufacturing process of a company in the state of Espirito Santo. The purpose is to evaluate which one of the process attributes correlate with the internal bond property, which is one of the requirements of ABNT standards 15316-2. After analyzing data from four months of production, it was found that the main worry refers to the production of two plate thicknesses, for $9 \mathrm{~mm}$ and $25 \mathrm{~mm}$. Pearson's correlation method indicated a subset of five attributes that highest affect the result, among the initial 23: percentage of glue, energy, speed and pressfactor and fiber moisture. Unlike other studies indicate, the high percentage of glue does not always guarantee the quality of the final product, leastwise for the $9 \mathrm{~mm}$ plate.
\end{abstract}

Resumo. Este artigo descreve a análise exploratória de dados do processo de fabricação de placas MDF de uma empresa no estado do Espírito Santo. O objetivo é avaliar quais dos atributos do processo estão correlacionados com a propriedade de resistência à tração perpendicular, que é um dos requisitos da norma ABNT 15316-2. Após analisar dados de quatro meses de produção, verificou-se que a preocupação é com a produção de duas espessuras de placas, para $9 \mathrm{~mm}$ e $25 \mathrm{~mm}$. O método de correlação de Pearson, indicou um subconjunto de cinco atributos que mais afetam o resultado, dentre as 23 iniciais: dosagem de resina, energia, velocidade e fator de prensagem e umidade da fibra. Diferente do que outros trabalhos indicam, nem sempre a alta dosagem de resina garante a qualidade do produto final, pelo menos para a placa de $9 \mathrm{~mm}$.

\section{Introdução}

Placas de MDF (Medium Density Fiberboard), ou painéis de fibra de média densidade são uma alternativa ao uso de madeira maciça como matéria-prima na fabricação de pisos e móveis. Como em outros processos na indústria, garantir a qualidade do produto final depende do controle e acompanhamento das variáveis produtivas. Como forma de padronizar e estabelecer valores mínimos de qualidade a painéis de fibra de média densidade, a Associação Brasileira de Normas Técnicas (ABNT) conta com a norma 15316-2 [ABNT 2015], que apresenta vários requisitos as propriedades físicas e mecânicas dos painéis: resistência à flexão estática, inchamento, resistência à tração e módulo de elasticidade.

A resistência à tração é um dos principais pontos examinados em laboratório para assegurar a qualidade do painel. Esse teste revela a resistência oferecida pela placa, até sua ruptura, ao ser submetida a uma força de tração de maneira perpendicular à sua superfície. O resultado da tração indicará a viabilidade de utilização do painel, conforme a Tabela 1. 
Tabela 1. Requisitos para painéis não estruturais para uso em condições secas. Fonte: ABNT (2015).

\begin{tabular}{|c|c|c|c|c|c|c|c|c|c|c|}
\hline \multirow{2}{*}{ Requisito } & & \multicolumn{7}{|c|}{ Critérios por faixa de espessura nominal (mm) } \\
\cline { 2 - 10 } & & $\begin{array}{c}1,8 \text { a } \\
2,5\end{array}$ & $\begin{array}{c}>2.5 \\
\text { a 4,0 }\end{array}$ & $\begin{array}{c}>4,0 \\
\text { a } 6,0\end{array}$ & $\begin{array}{c}>6,0 \\
\text { a 9,0 }\end{array}$ & $\begin{array}{c}>9,0 \\
\text { a } \\
12,0\end{array}$ & $\begin{array}{c}>12 \text { a } \\
19,0\end{array}$ & $\begin{array}{c}>19,0 \\
\text { a } 30\end{array}$ & $\begin{array}{c}>30,0 \\
\text { a 45,0 }\end{array}$ & $\begin{array}{c}> \\
45,0\end{array}$ \\
\hline $\begin{array}{c}\text { Resistência à tração } \\
\text { perpendicular (mínimo) }\end{array}$ & $\begin{array}{c}\mathrm{N} / \mathrm{m} \\
\mathrm{m}^{2}\end{array}$ & 0,65 & 0,65 & 0,65 & 0,65 & 0,60 & 0,55 & 0,55 & 0,50 & 0,50 \\
\hline
\end{tabular}

Caso os resultados laboratoriais indiquem valores abaixo do mínimo definido pela norma, significa que é necessária uma adequação do processo visando enquadrá-lo à norma. Segundo Eleotério (2000), vários trabalhos analisaram de forma isolada os efeitos causados às propriedades físicas e mecânicas do MDF por alguns fatores do processo e da matéria-prima. Este trabalho faz a análise exploratória dos dados de produção de uma fábrica de MDF a fim de compreender quais dos atributos coletados poderiam revelar insights, resultando na melhoria do produto e do custo.

\section{Materiais}

Linhas de produção de painéis de média densidade podem variar em determinadas etapas, contudo, assemelham-se ao fluxo apresentado na Figura 1. O processo é iniciado pela entrada de toras em um fragmentador resultando em partículas ou cavacos de tamanhos semelhantes. Os cavacos são classificados por peneiras, cozidas e armazenados em silos. As fibras são obtidas do cavaco através de desfibradores mecânicos, recebendo adição de resina, em seguida são levadas para formação de uma espécie de colchão que atenderá a uma espessura e densidade pré-determinada. O colchão passará por uma pré prensa que tem objetivo de diminuir a altura e melhorar sua consistência e por fim a manta é submetida a uma prensagem contínua. Posteriormente são submetidas a um processo de secagem (resfriamento e climatização), lixamento e embalagem [Iwakiri et al 2005].

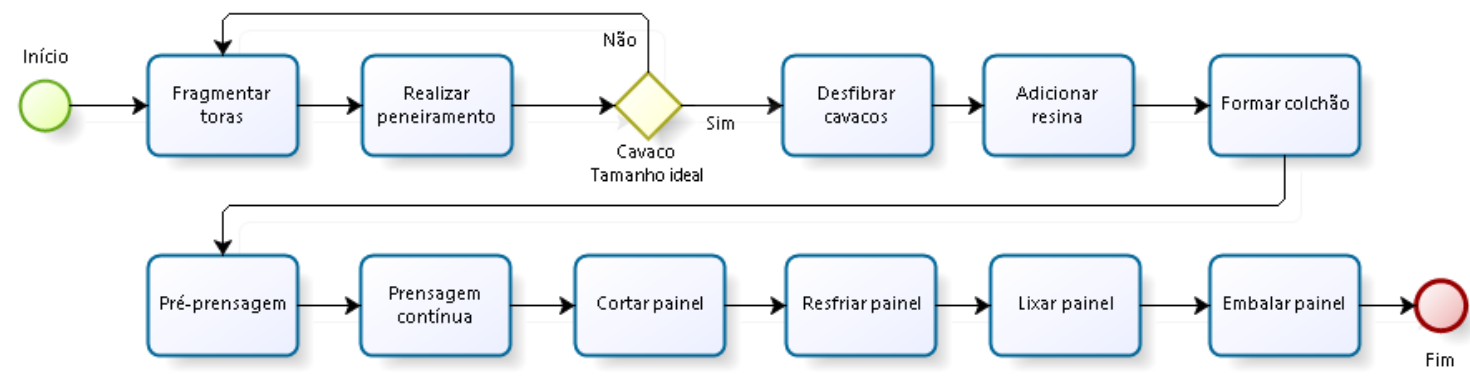

Figura 1. Processo industrial de produção de MDF. Elaborado pelo autor, inspirado em Ilan (2019).

Foram coletados os dados de produção de uma empresa do estado do Espírito Santo, durante o período de 23/04/2019 a 20/08/2019, resultando em 681 linhas de informação, onde cada linha representa a fabricação de uma placa. Ao total foram 23 atributos coletados durante o processo de produção: data, hora, espessura da placa, pressão do desfibrador, pressão do digestor, tempo de cozimento, energia específica (energia gasta por tonelada de fibra refinada), tipo de resina, dosagem de resina (percentual de cola aplicado no painel), fornecedor de resina, dosagem de parafina (percentual de emulsão aplicado no painel), concentração manifold (diluição da resina com água), offset pressão de cola (pressão no bico injetor de resina), offset pressão de vapor (pressão de vapor na aplicação da resina), umidade 
da fibra, densidade da fibra, quantidade de spray dosado por cima do colchão, quantidade de spray dosado na cinta de formação abaixo do colchão, concentração de desmoldante diluída no spray, temperatura da pré-compressão, velocidade que placa de MDF passou pela esteira, fator de prensagem (tempo que cada metro do painel fica na prensa) e sobre espessura (espessura extra para ser retirada pela lixadeira).

Além destes, há a informação do resultado dos testes laboratoriais, que são as medidas de sete pontos de tração realizados ao longo da placa teste. A média destes valores, gera a tração média usada na comparação da Tabela 1 . Caso a tração média esteja abaixo do mínimo, indica que a placa produzida não atendeu a norma de qualidade.

\section{Resultados e Discussão da Análise Exploratória}

De acordo com Batista (2003), considera-se que a análise exploratória de dados é um processo semi-automático, isto é, depende da capacidade da pessoa que a conduz em identificar os problemas presentes nos dados, e utilizar os métodos mais apropriados para solucionar cada um dos problemas. Este trabalho foi conduzido desta forma, para a análise dos dados foi utilizada a linguagem Python com as bibliotecas numpy, Pandas, matplotlib e seaborn.

Foram calculadas várias medidas estatísticas e vários gráficos foram gerados. Serão apresentados apenas os gráficos que apresentaram resultados mais significativos. Como primeiro passo, foi feita a avaliação dos resultados de qualidade pela espessura. Não houve nenhuma placa rejeitada na espessura de $6 \mathrm{~mm}$. Na Figura 2, apresenta-se o gráfico do percentual de placas de acordo com a norma e fora da norma, de acordo com a espessura da placa $(9,12,15,18$ e $25 \mathrm{~mm})$. A empresa manifestou preocupação com as placas fora da norma para as placas de 9 e $25 \mathrm{~mm}$, que percentualmente indicam que quase metade da produção dessas espessuras apresentaram problemas de qualidade.

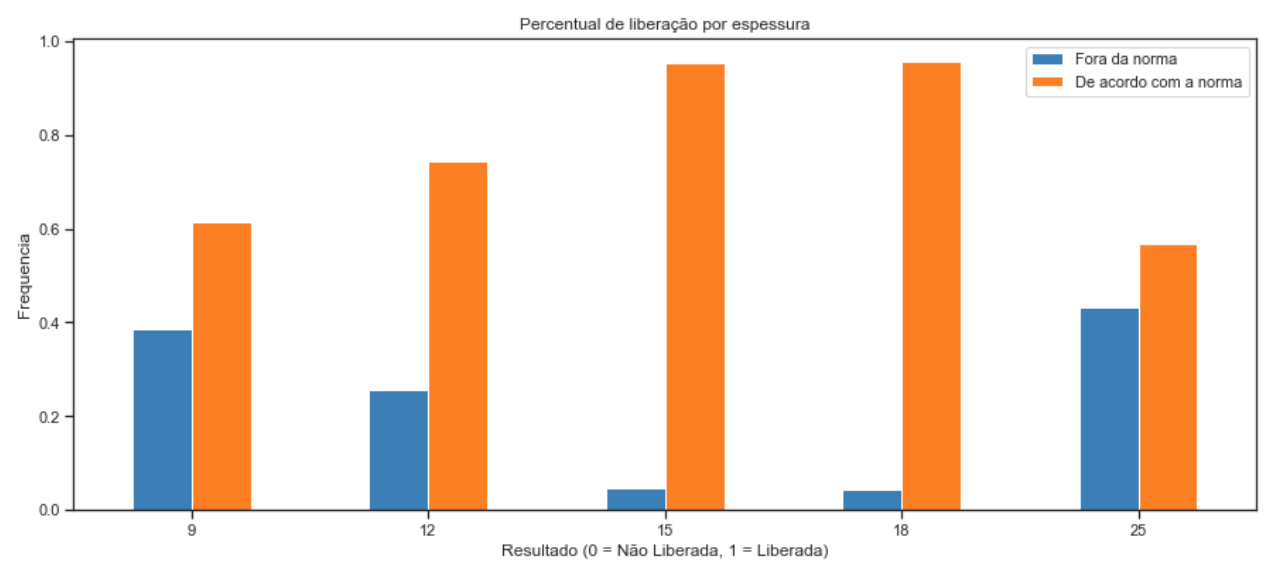

Figura 2. Gráfico do percentual de placas de acordo com a norma e fora da norma, de acordo com a espessura.

Conforme Faceli et al. (2015) uma forma de reduzir a quantidade de atributos a serem examinados é identificar e selecionar os mais relevantes. Para isso, foram selecionados os que apresentaram maior coeficiente de correlação, positiva ou negativa, em relação ao atributo resultado. O método de correlação utilizado foi o de Pearson, e resultaram nos seguintes atributos: dosagem de resina, energia específica, velocidade e fator de prensagem e umidade da fibra. As Figuras 3 e 4 apresentam gráficos pairplot destes 5 atributos para as 
espessuras de 9 e $25 \mathrm{~mm}$, respectivamente. Analisando os gráficos, percebe-se que os painéis de 9 e $25 \mathrm{~mm}$ não compartilham de mesmo cenário.
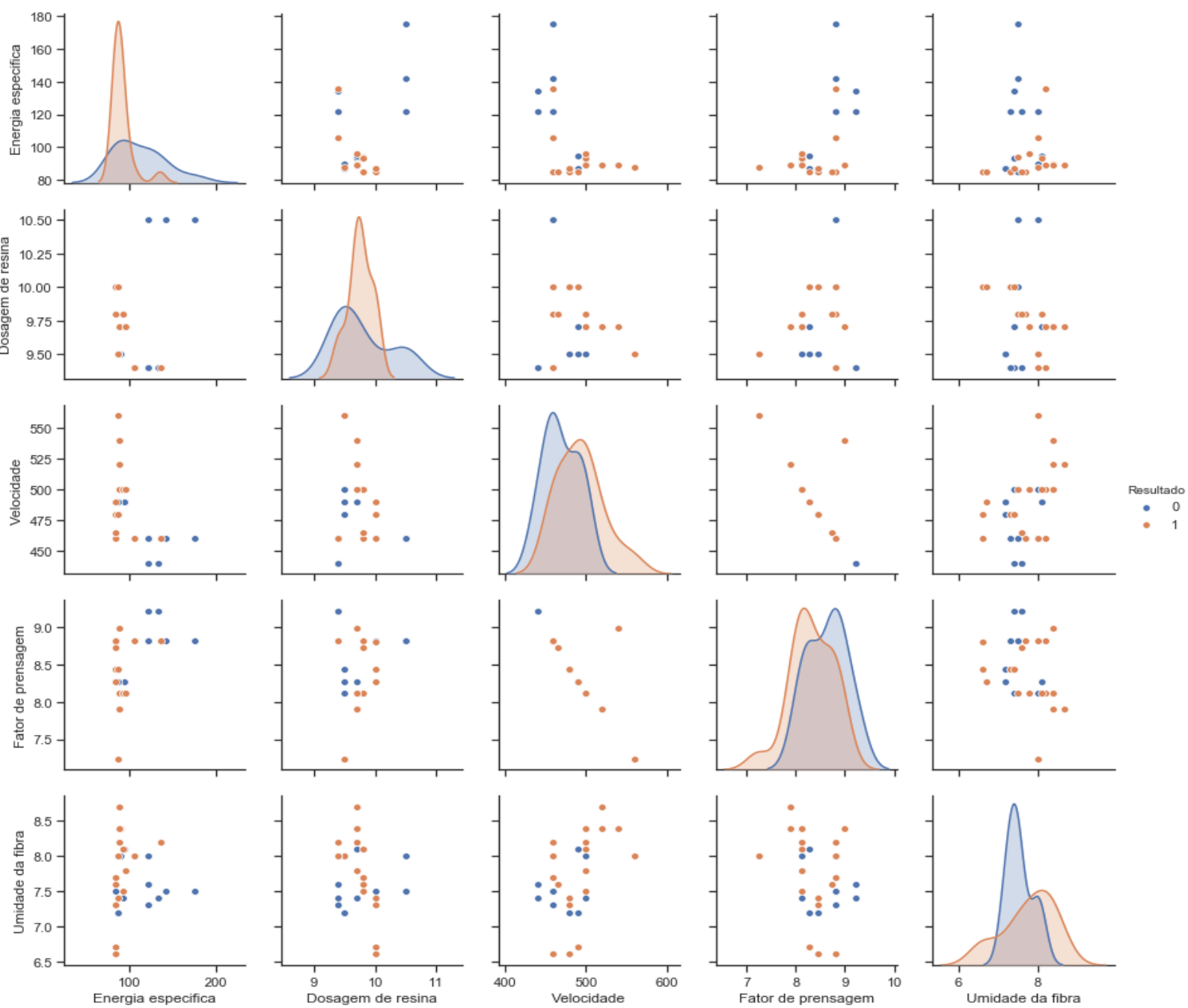

Figura 3. Gráfico pairplot dos atributos mais correlacionados, energia específica, dosagem de resina, velocidade, fator de prensagem e umidade da fibra para espessura de de $9 \mathrm{~mm}$.

Para a espessura de $9 \mathrm{~mm}$, peças fora da norma apresentam alto valor de energia específica. Pelo gráfico, o alto valor de energia está correlacionado com a alta dosagem de resina, baixa velocidade e alto fator de prensagem. É possível observar que ocorrem problemas com a placa para uma alta dosagem de resina $(10,5)$, e para um alto fator de prensagem (acima de $9,0)$. Quando ocorre conjuntamente um alto fator de prensagem com baixa velocidade, a placa finaliza fora da norma. Não se encontrou correlação com a umidade da fibra.

Já para a espessura de $25 \mathrm{~mm}$, não se encontrou correlação com a energia específica. Os problemas ocorrem com alta velocidade, ao contrário do que ocorre para a espessura de $9 \mathrm{~mm}$. Quando ocorre conjuntamente alta velocidade com valor médio de fator de prensagem (em torno de 15), a placa finaliza fora da norma. Para este valor médio de fator de prensagem, independente dos outros atributos, a placa fica fora da norma. Para valores baixos de dosagem de resina (menor que 9,5) as placas apresentam problemas, o que também ocorreu para valores altos de umidade. 

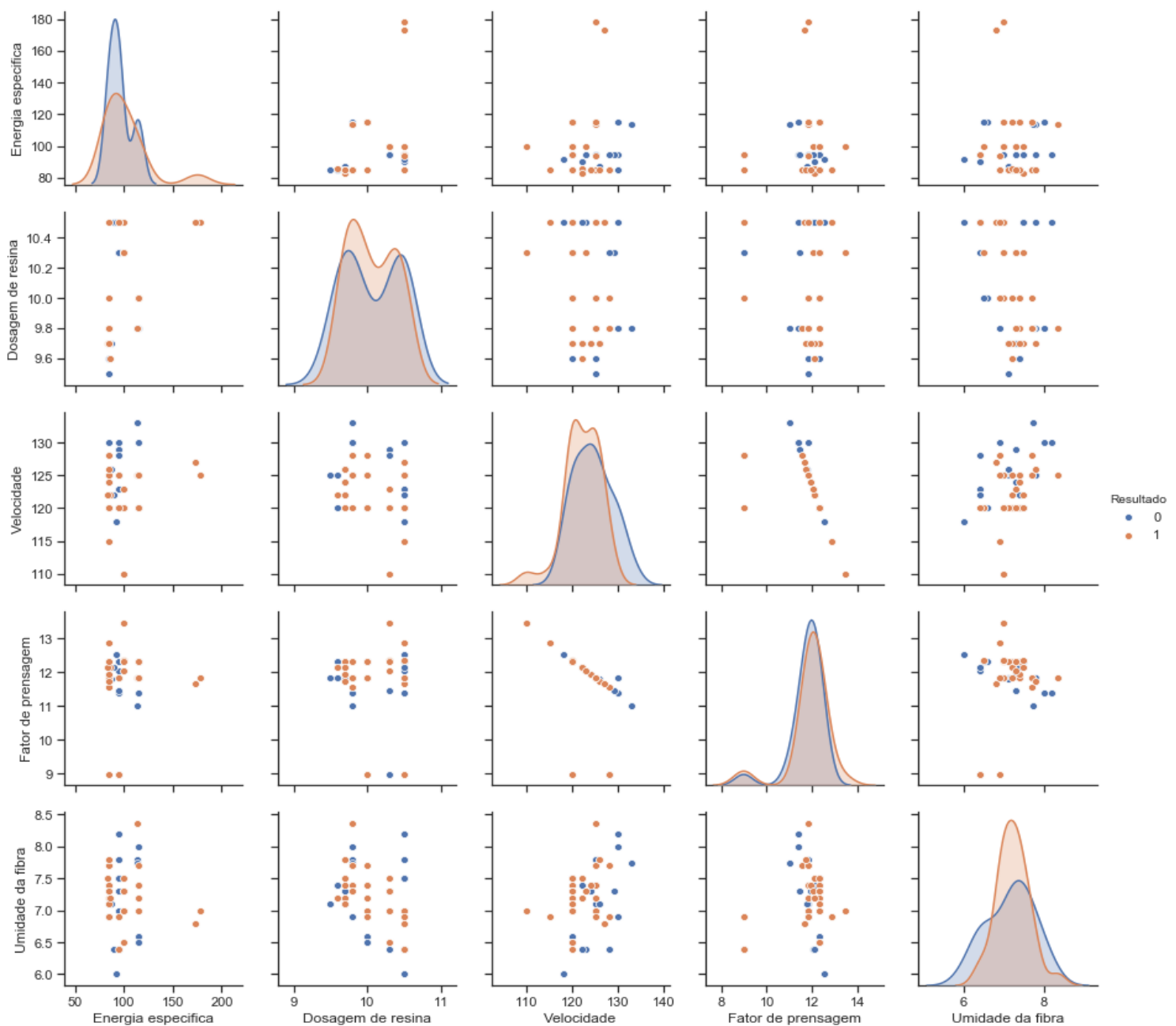

Figura 4. Gráfico pairplot dos atributos mais correlacionados, energia específica, dosagem de resina, velocidade, fator de prensagem e umidade da fibra para espessura de $25 \mathrm{~mm}$.

É possível se traçar um paralelo com o trabalho de Campos (2003), que afirma que quando o teor de umidade é elevado ocorre aumento no ciclo de prensagem porque é necessário um maior tempo para a retirada da umidade excedente do painel. Quando o teor de umidade é muito reduzido ocorre um perfil de densidade mais homogêneo e, consequentemente, uma maior resistência à tração perpendicular. Este efeito da umidade foi encontrado na espessura de $25 \mathrm{~mm}$, mas não foi encontrada na de $9 \mathrm{~mm}$.

Campos (2003) também define que a resistência à tração é afetada pelo fator de prensagem. Esta correlação foi encontrada na espessura de $9 \mathrm{~mm}$, para alto valor de prensagem há problemas com a placa. No entanto, para a placa de $25 \mathrm{~mm}$, o valor médio deste fator é que está mais correlacionado com problemas na tração. Só quando o fator de prensagem está associado à baixa velocidade da prensa é que resulta em problemas de tração no resultado.

A energia específica para a espessura de 9mm indica que o tempo da exposição do cavaco na fase de desfibramento consome maior energia e com isso reduz as propriedades mecânicas e físicas, mesmo com alto teor de resina.

Um dos maiores custos da produção de MDF é dado pela resina. Segundo Iwakiri (2005), por representar até $35 \%$ do custo de produção, assim, é importante otimizar a quantidade de 
resina no processo. Campos e Lahr (2004) sugeriram produzir painéis utilizando adesivo à base de uréia-formaldeído visando manter adequada as propriedades dos painéis, tais como a tração perpendicular, reduzindo o teor de adesivo e consequentemente o custo de produção. Castro (2000) também avalia a economia do processo e que a qualidade do MDF, são resultantes de uma adequada aplicação da resina. Nestes trabalhos, indica-se que painéis com alto teor de resina são mais rígidos e resistentes. No entanto, os dados mostraram que para a espessura de $9 \mathrm{~mm}$, ter um alto teor de resina resultou em um efeito contrário, a alta dosagem de resina (10,5\%), apresentada pela Figura 3, não garantiram boa qualidade. Os gráficos evidenciam que o não atendimento a norma pode estar atrelado a outros atributos como velocidade, energia específica e fator de prensagem.

\section{Conclusão}

Processos industriais são suscetíveis aos insumos utilizados, aos equipamentos, e a cada parâmetro de cada etapa. A análise exploratória dos dados industriais da fábrica de painéis de MDF apresentou bons insights acerca do problema de qualidade enfrentado na produção das placas de 9 e $25 \mathrm{~mm}$.

A identificação do comportamento distinto entre alguns atributos ao cumprimento da norma pode ser considerado um resultado interessante. Ele sugere que os ajustes, buscados na melhoria do custo e da qualidade, ao se produzir painéis de média densidade, não devem ser genéricos e sim individualizados pela espessura da placa. Espera-se que este trabalho tenha continuidade visando a melhoria contínua da qualidade de produção de painéis, bem como diminuição dos custos da produção. Como trabalho futuro, planeja-se usar técnicas de aprendizado de máquina para a predição da qualidade do MDF.

\section{Referências}

ABNT - Associação Brasileira de Normas Técnicas. (2015). NBR 15316-2: painéis de fibras de média densidade. Parte 2: requisitos e métodos de ensaio. Rio de Janeiro.

Batista, G. E. A P. A. (2003). Pré-processamento de dados em aprendizado de máquina supervisionado. Orientadora: Maria Carolina Monard. 2003. 204 f. Tese (Doutorado em Ciências) - Universidade de São Paulo.

Campos, C. I. (2003). Processo produtivo de chapa de fibra de média densidade (MDF). Revista da Madeira, ed. 71, maio.

Campos, C. I.; LAHR, F. A. R. (2004). Estudo Comparativo dos Resultados de Ensaio de Tração Perpendicular para MDF Produzido em Laboratório com Fibras de Pinus e de Eucalipto Utilizando Uréia-Formaldeído. Revista Matéria, 9(1):29-40.

Castro, E. M. (2000). Estudo da usinabilidade de chapas MDF (Medium Density Fiberboard) para usinagem de desbaste e acabamento. São Carlos. Dissertação (M.S.) - Escola de engenharia de São Carlos, Universidade de São Paulo.

Eleotério, J. R. (2000). "Propriedades físicas e mecânicas de painéis MDF de diferentes densidades e teores de resina". Piracicaba. Dissertação (M.S.) - Escola Superior de Agricultura "Luiz de Queiroz", Universidade de São Paulo.

Faceli, K; Lorena, A. C.; Gama, J.; Carvalho, A. C. P. L. F. (2015). Inteligência Artificial Uma Abordagem de Aprendizado de Máquinas. LTC.

Iwakiri, S. (2005). Painéis de madeira reconstituída. Curitiba: FUPEF. 247p. 